\title{
Lumbar Incisional Hernia Repair following Iliac Bone Graft Harrest
}

Ketan Vagholkar', Abhifit Budhkar ${ }^{2}$, Jagruti Gulati²

From the Dr.D.Y.Patil Medical College', Navi Mumbai, Maharashtra;

Rajawadi Municipal General Hospital2 , Mumbai, Maharashtra, India.

\section{Abstract:}

Background: Lumbar incisional hernia following iliac bone grafting poses an anatomical challenge to the attending surgeon. Alteration of the local anatomy necessitates meticulous preoperative evaluation and proper planning of the repair. Introduction: The incidence of such incisional hernias is between $5-7 \%$. The chances of complications are high. Case Report: A case of lumbar incisional hernia following iliac bone graft harvesting is presented. Discussion: The planning and technical considerations of surgical repair are discussed. Conclusion: Pre-operative CT scan evaluation of the hernia followed by open mesh repair is advisable as it ensures safety and offers good results.

Key words: Hernia, Herniorrhaphy, Lumbosacral Region, Bone Transplantation, lleum, Humans.

\section{Introduction}

Iliac crest is the commonest site for harvesting autogenous bone grafts. A multitude of complications including incisional hernia have been reported. Lumbar incisional hernia developing through a scar of iliac crest bone graft harvesting is relatively uncommon with a reported incidence of $5-7 \%$ [1]. Complications such as incarceration and strangulation are likely to cause high morbidity [2]. A case of lumbar incisional hernia following iliac bone graft harvesting repaired by polypropylene hernioplasty is reported along with review of literature to highlight the surgical challenge it poses.

\section{Case Report}

A 40 year old male patient presented with swelling underlying a scar of iliac crest bone graft harvest surgery. Patient had sustained a vehicular accident during which he had sustained a fracture of the right tibia. Due to delayed fracture healing, a revision surgery including a bone grafting was done by the attending orthopedic surgeon. The iliac wound healed primarily without any complications. The long bone fracture also healed with good physical rehabilitation of the patient. Four months after the revision orthopedic surgery the patient noticed a swelling underlying the iliac scar [Fig.1]. Physical examination of the swelling exhibited a visible and palpable impulse on coughing. Contrast enhanced CT scan was done and revealed a defect at the site of the iliac bone graft harvest. The contents of sac were intestinal loops including the right colon [Fig.2].

\section{Corresponding Author: Dr. Ketan Vagholkar}

Email: kvagholkar@yahoo.com

Received: July 22, 2014 | Accepted: September 2, 2014 | Published Online: October 5, 2014

This is an Open Access article distributed under the terms of the Creative Commons Attribution License (creativecommons.org/licenses/by/3.0)

Conflict of interest: None declared | Source of funding: Nil | DOl: http://dx.doi.org/10.17659/01.2014.0096 
Patient underwent surgical intervention wherein open meshplasty was performed. The sac was dissected up to the neck and opened. The contents were cecum with appendix and terminal ileum [Fig.3]. The contents were reduced and a herniotomy performed with 3-0 silk. The edges of defect were clearly delineated and a polypropylene mesh was placed and was fixed to the surrounding muscles with 2-0 prolene. The overlying muscle sheet which was lying detached from the iliac crest was undermined superiorly and approximated to the iliac crest. Post-operative recovery was uneventful with excellent wound healing.

Patient has been following up for the last 2 months without any evidence of recurrence [Fig. 4].

\section{Discussion}

The lumbar region of the human body has a complex anatomy with respect to various muscle attachments

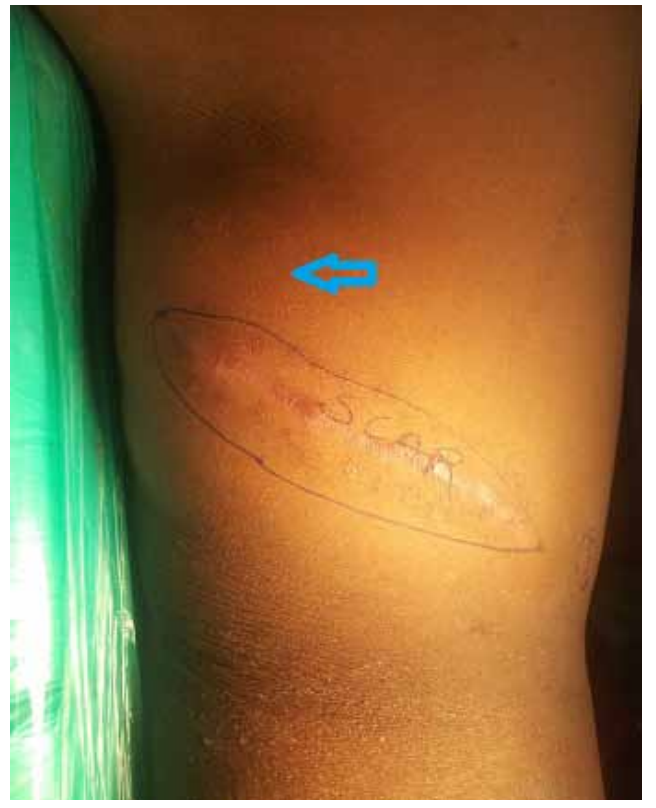

Fig.1: Preoperative photograph showing the incisional hernia (blue arrow points to the hernia swelling). and fascias. The traditionally described superior and inferior lumbar triangles are potential areas of weakness by virtue of intricate relationships of fascia, muscles and bone. Congenital hernia can develop through these triangles in a patient with muscular hypoplasia as seen in lumbocostovertebral syndrome [3]. However, acquired hernias in this region are associated with severe weakening of the muscles. This may be intrinsic as in malnourished individuals or extrinsic as seen in incisional hernias. Development of lumbar incisional hernia through the inferior lumbar triangle following iliac crest bone graft harvest has been described in literature. Review of literature on PubMed and Google Scholar reveals not more than 400 cases reported till date.

Various factors may contribute to the development of these hernias. Bone graft harvesting by itself creates a potential bony defect [Fig.5]. The musculoaponeurotic structures get detached during

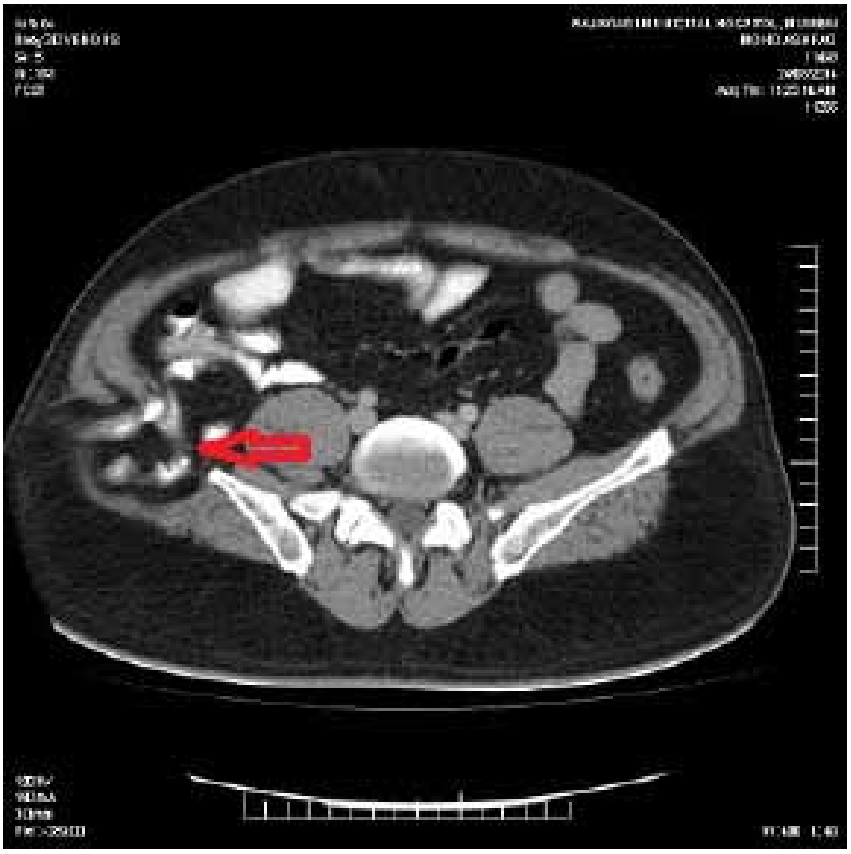

Fig.2: CT scan showing the defect and the contents of the hernia sac marked by the red arrow. 


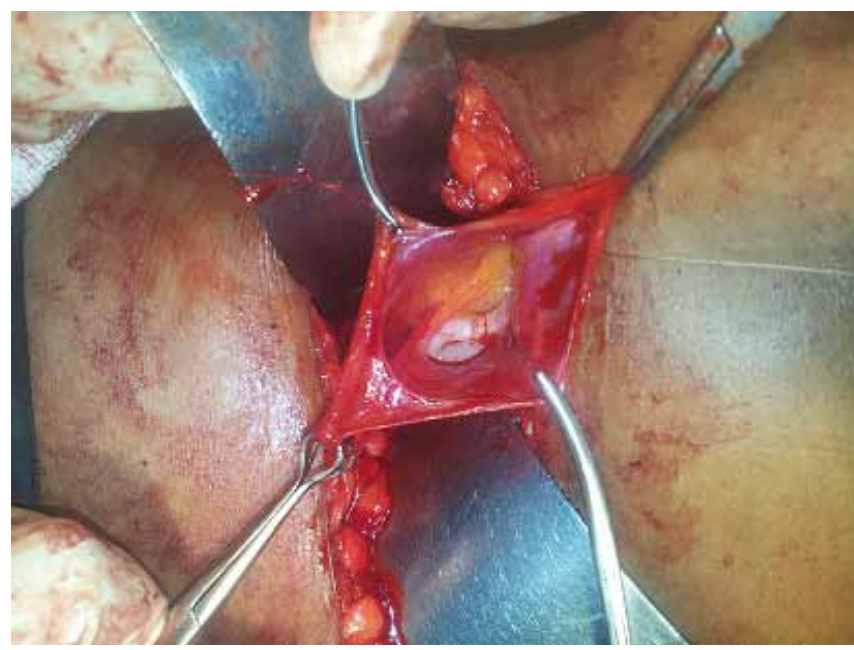

Fig.3: Intraoperative photograph showing the opened sac with appendix and the caecum as contents.

the course of the procedure and remain unsupported despite sutured approximation. However, due to the tension on the non-elastic musculoaponeurotic structures the chances of give way are very high thereby giving rise to a defect resulting in an incisional hernia. The contents of such incisional hernias can vary from loops of small intestine to the cecum and ascending colon as seen in the case presented. Therefore great care and precision needs to be exercised while dealing with the sac and its contents.

Contrast enhanced CT scan of the abdomen is necessary for an accurate preoperative evaluation of the case with respect to the exact location and size of the defect [4]. The detailed status of the contents is also revealed. Several methods have been described to repair such lumbar incisional hernias, including bone transfer and various soft tissue flaps. The Bosworth repair describes transferring the anterior superior iliac spine inferiorly and posteriorly, drawing muscle and fascia over the defect [5]. The Dowd technique advocates the use of a pedunculated gluteal fascia flap to cover the defect [6]. The Koontz technique involves a lumbar

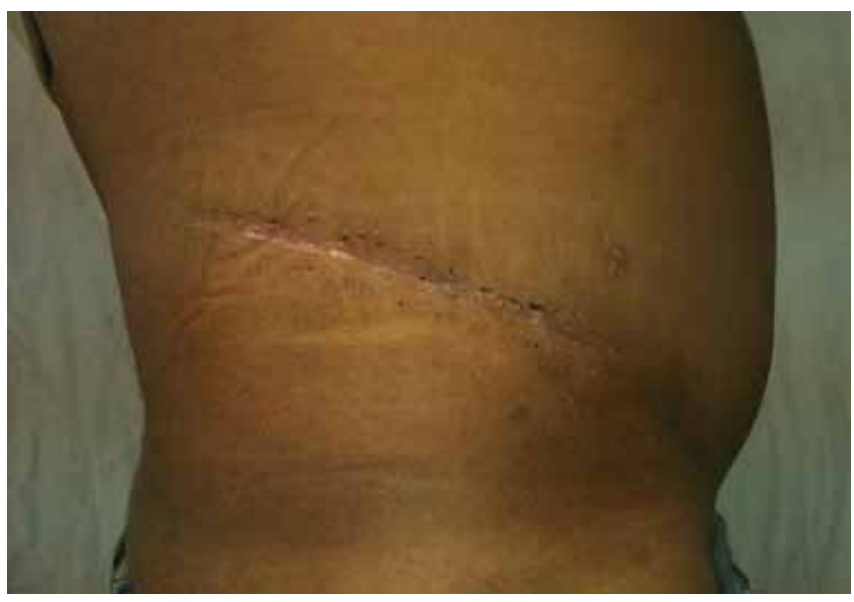

Fig.4: Postoperative state.

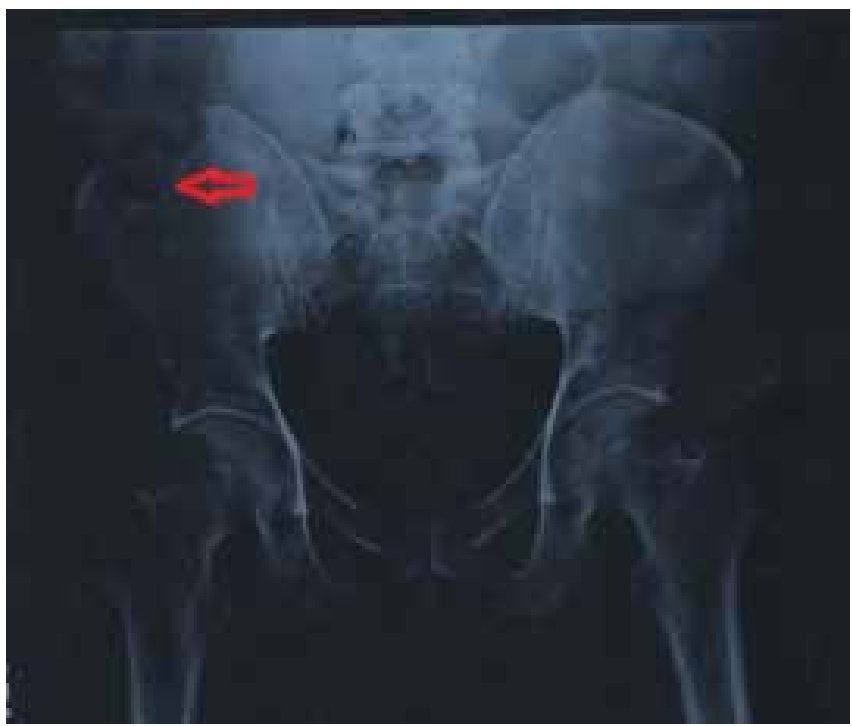

Fig.5: X-ray of the pelvis showing the defect in the right iliac crest marked by a red arrow from where the bone grafts were harvested.

fascia flap for repair [6]. Laparoscopic approach has also been described in the literature [7]. But in a situation of the cecum and ascending colon being involved this could prove to be dangerous as dissecting the contents could inevitably lead to damage to the colon. Open approach therefore continues to be the safest for such hernias. As there are a lot of dynamic forces acting continuously on the 
muscles attached to the iliac crest, the detachment of these musculoaponeurotic structures from a portion of iliac crest will lead to shortening of the length of affected muscles with time thereby creating a large defect. This explains the rapid increase in the size of hernia with time. Therefore repair is advised at an early stage approximately by 6 months after primary surgery. Attempts to undermine the surrounding contracted muscles proves to be futile in majority of cases especially in cases wherein the surgery is delayed. A wide mesh repair is therefore a procedure of choice. Repair with a synthetic mesh is advisable in most of the cases. [8]

Utmost care needs to be taken that the mesh extends for at least 1 inch beyond the defect under the overlying adjacent muscles all around the circumference. The use of suture bone anchors or corkscrew anchors to fix the mesh have been described [9]. The mesh can also be secured to overlying muscle and the periosteum with 2-0 prolene as done in the case presented. A negative suction drain is placed over the mesh in order to prevent the development of a seroma. Negative suction drain also helps in obliterating the dead space thereby enhancing snug approximation between the musculoaponeurotic layer and the mesh. Post-operative care plays a significant role in the outcome of such patients. Patient is advised not to strain or perform any physical activity which puts additional stress at the operated site for at least 6 months until the process of maturation of fibrous tissue is completed.

\section{Conclusion}

Lumbar incisional hernia following bone graft harvesting from the iliac crest poses an anatomical challenge to the operating surgeon. Preoperative CT scan evaluation provides an excellent road map for planning the repair. Early open repair is advisable as it allows meticulous identification and safe dissection of contents at time of herniotomy. A mesh repair provides adequate strength thereby preventing recurrence.

\section{References}

1. Fowler BL, Dall BE, Rowe DE. Complications associated with harvesting autogenous iliac bone graft. Am J Orthop (Belle Mead NJ) 1995;24(12):895-903.

2. Velchuru VR, Satish SG, Petri GJ, Sturzaker HG. Hernia through an iliac crest bone graft site: report of a case and review of the literature. Bull Hosp Jt Dis 2006;63(3-4):166-168.

3. Vagholkar K, Dastoor K. Congenital lumbar hernia with lumbocostovertebral syndrome: A case report and review of the literature. Case reports in pediatrics. Vol 2013, Article ID 53290 4 pages 2013, doi:10.1155/2013/532910.

4. Guillem P, Czarnecki E, Duval G, Bounoua F, Fontaine $C$. Lumbar hernia: anatomical route assessed by computed tomography. Surg Radiol Anat 2002;24(1):53-56.

5. Bosworth DM. Repair of herniae through iliaccrest defects. J Bone Joint Surg Am 1955;37-A (5): 1069-1073.

6. Di Carlo I, Toro A, Sparatore F, Corsale G. Lumbar hernia repaired using a new technique. Am Surg 2007;73(1):54-57.

7. Yurcisin BM, Myers CJ, Stahlfeld KR, Means JR. Laparoscopic hernia repair following iliac crest harvest. Hernia 2010;14(1):93-96.

8. Auleda J, Bianchi A, Tibau R, Rodriguez-Cano $O$. Hernia through iliac crest defects. A report of four cases. Int Orthop 1995;19(6):367-369.

9. Carbonell AM, Kercher KW, Sigmon L, et al. A novel technique of lumbar hernia repair using bone anchor fixation. Hernia 2005;9(1):22-25. 